\title{
DE L'EXPOSITION UNIVERSELLE À LA FOIRE NATIONALE. ÉLÉMENTS POUR UNE HISTOIRE ÉLARGIE DES EXPOSITIONS DURANT LA PREMIERE GUERRE MONDIALE
} Claire-Lise Debluë

Presses Universitaires de France | «Relations internationales »

2015/4 $n^{\circ} 164 \mid$ pages 59 à 74

ISSN 0335-2013

ISBN 9782130651208

Article disponible en ligne à l'adresse :

http://www.cairn.info/revue-relations-internationales-2015-4-page-59.htm

\section{Pour citer cet article :}

Claire-Lise Debluë, «De l'exposition universelle à la foire nationale. Éléments pour une histoire élargie des expositions durant la Première Guerre mondiale », Relations internationales 2015/4 ( $\mathrm{n}^{\circ} 164$ ), p. 59-74.

DOI 10.3917/ri.164.0059

Distribution électronique Cairn.info pour Presses Universitaires de France.

(C) Presses Universitaires de France. Tous droits réservés pour tous pays.

La reproduction ou représentation de cet article, notamment par photocopie, n'est autorisée que dans les limites des conditions générales d'utilisation du site ou, le cas échéant, des conditions générales de la licence souscrite par votre établissement. Toute autre reproduction ou représentation, en tout ou partie, sous quelque forme et de quelque manière que ce soit, est interdite sauf accord préalable et écrit de l'éditeur, en dehors des cas prévus par la législation en vigueur en France. Il est précisé que son stockage dans une base de données est également interdit. 


\title{
De l'exposition universelle à la foire nationale. Éléments pour une histoire élargie des expositions durant la Première Guerre mondiale
}

\begin{abstract}
Depuis quelques années, on assiste à une orientation nouvelle des "expositions" dans un sens plus pratique, vers des réalités plus immédiates et tangibles. Avant la guerre déjà, la lassitude des expositions s'emparait de plus en plus des industriels; ils constataient que les résultats ne correspondaient pas toujours aux dépenses et aux peines. L'exposition, en effet, attirait les curieux, mais combien d'entr'eux (sic) devenaient des clients? Beaucoup venaient tout simplement visiter, mais non point dans l'idée d'acheter plus tard'.
\end{abstract}

L'éclatement de la Première Guerre mondiale marque un point de rupture incontestable avec les pratiques antérieures de l'exposition. Jusqu'en 1925 et l'Exposition internationale des arts décoratifs et industriels modernes de Paris, aucun événement d'une envergure comparable aux expositions universelles n'a lieu en Europe. La disparition soudaine et durable de ces manifestations, telles qu'elles avaient été conçues depuis la Great Exhibition of the Works of Industry of all Nations organisée à Londres en 1851, fait place à l'expression de nouvelles conceptions de l'organisation commerciale, ajustées aux impératifs de l'économie de guerre. Si la diversification des acteurs de la politique étrangère favorise l'apparition de bureaux de renseignements commerciaux ${ }^{2}$, certaines organisations dédiées à l'ouverture des débouchés et au développement d'industries nouvelles,

1. L.[ouis] B.[éguin], «Expositions, Foires et Comptoirs ", Revue Économique, 10 mai 1917 [s.p.].

2. Laurence Badel, "Pour une histoire de la diplomatie économique de la France ", Vingtième Siècle. Revue d'histoire, n 90, vol. II, 2006, p. 170. 
fonctionnant comme de véritables services d'informations, voient également le jour : les foires d'échantillons. Dès 1914, et à plus forte raison dans l'immédiat après-guerre, la foire dite moderne est consacrée comme le nouveau paradigme de l'exposition commerciale. Quelle était la vocation des foires ? Dans quelle mesure s'écartaient-elles du modèle historique de l'exposition universelle et jusqu'à quel point témoignaient-elles de l'émergence d'un "ordre économique nouveau ${ }^{3}$ " ?

En dépit du nombre considérable d'études consacrées aux expositions universelles, l'historiographie n'a accordé qu'une attention limitée aux foires commerciales qui, dès 1914, se multiplient en Europe $e^{4}$. Les foires dites modernes représentent pourtant bien davantage qu'un épiphénomène de la Première Guerre mondiale. Elles sont en effet emblématiques de reconfigurations plus importantes que l'on situera à la croisée de deux préoccupations : la centralisation et la concentration des organisations industrielles et commerciales d'un côté, l'institutionnalisation et la professionnalisation du champ publicitaire de l'autre .

Afin de comprendre à quelles conditions le principe de la foire commerciale s'est imposé durant la Première Guerre mondiale, nous reviendrons, dans un premier temps, sur les enjeux transnationaux de la politique d'exposition au tournant du $\mathrm{Xx}^{\mathrm{e}}$ siècle et sur la perte d'influence du modèle historique de l'exposition universelle. Cette brève mise en contexte nous conduira à aborder les principales caractéristiques des foires d'échantillons européennes et leur fonction dans l'économie de guerre. En nous arrêtant plus particulièrement sur le cas suisse et sur la création, en 1916, d'une foire nationale d'échantillons, nous montrerons qu'au-delà des particularismes régionaux qui la caractérisent, cette manifestation s'inscrit dans un réseau transnational des foires qui contribuera, au sortir de la guerre, à la restauration d'un "internationalisme libéral ${ }^{6} »$. Nous conclurons enfin cet article en évoquant les ajustements des pratiques publicitaires de la foire aux conditions nouvelles de l'organisation commerciale.

3. L. G., "De l'utilité des Foires d'échantillons », La Foire suisse d'échantillons. Bulletin officiel, n 2, 15 mars 1922, p. 26.

4. Sur les expositions internationales, voir la bibliographie des études parues jusqu'en 2005, établie par Alexander C.T. Geppert, Jean Coffey et Tammy Lau, International Exhibitions, Expositions Universelles and World's Fairs, 1851-2005: A Bibliography, Freie Universität Berlin, Allemagne, California State University, Fresno, États-Unis, [s.d.], pp. 1-94. Sur les foires commerciales, voir les différentes contributions réunies dans l'ouvrage édité par Hartmut Zwahr, Thomas Topfstedt et Günter Bentele, Leipzigs-Messen, 1497-1997, Cologne/Weimar/Vienne, Böhlau Verlag, vol. II, 1999, ainsi que l'article de Stephen Gross, "Selling Germany in South-Eastern Europe: Economic Uncertainty, Commercial Information and the Leipzig Trade Fair 1920-40 ", Contemporary European History, n 1, vol. XXI, février 2012, pp. 19-39. Pour la période suivant la Deuxième Guerre mondiale, les travaux sont en revanche beaucoup plus nombreux.

5. J. Donald Shearer, "The Reichskuratorium für Wirtschaftlichkeit: Fordism and Organized Capitalism in Germany, 1918-1945 ", The Business History Review, n 4, vol. LXXI, hiver 1997, pp. 569-602 ; Marie-Emmanuelle Chessel, La Publicité, naissance d'une profession (1900-1940), Paris, CNRS Éditions, 1998, pp. 20 sq.

6. Michel Fior, Institution globale et marchés financiers. La Société des Nations face à la reconstruction de l'Europe, 1918-1931, Berne, Peter Lang, 2008, p. 111. 


\section{L'EXPOSITION UNIVERSELLE CONTESTÉE : «FATIGUE DES EXPOSITIONS » ET CRISE DU LIBÉRALISME MANCHESTÉRIEN}

Pour comprendre de quelle manière le modèle de la foire s'est imposé durant la guerre et dans l'immédiat après-guerre, il convient de revenir brièvement sur les résistances suscitées, dès les années 1870, par le modèle libre-échangiste de l'exposition universelle. Dans le contexte de la Grande Crise et face aux politiques protectionnistes adoptées par les puissances industrielles européennes, le principe historique de l'exposition universelle réunit un grand nombre de détracteurs issus des rangs-mêmes des exposants. Dans les rapports adressés par les commissaires généraux à leurs gouvernements ou par voie de presse, ce phénomène est couramment qualifié de "fatigue des expositions" 7 . Face à la nécessité de conquérir de nouvelles positions sur les marchés extérieurs et de se distinguer de leurs concurrents, certains des secteurs les plus importants de l'industrie d'exportation, comme celui de l'industrie alimentaire, mobilisent d'autres canaux d'information, jugés plus efficaces et moins onéreux ${ }^{8}$. Au tournant du siècle, les expositions universelles ont perdu une partie de leur attrait, si bien que de nombreux observateurs estiment que celles-ci entonnent leur "chant du cygne ${ }^{9}$ ".

Malgré certaines tentatives de réglementation, le climat de défrance à l'égard des expositions universelles se prolonge jusqu'au début du $\mathrm{XX}^{\mathrm{e}}$ siècle. Il faut ainsi attendre 1906 pour que le projet d'une réglementation internationale des expositions aboutisse enfin. Réunies en assemblée lors de l'Exposition internationale de Milan, les chambres de commerce approuvent la mise sur pied d'une institution ayant pour mandat de réguler le nombre, la nature et la fréquence des expositions ${ }^{10}$. La réglementation des récompenses, des procédures de nomination des jurys, et de la protection industrielle constitue la pierre angulaire de cette entente internationale, qui s'institutionnalise en 1907 avec la création de la Fédération internationale des comités permanents d'expositions (FICPE). La rédaction d'une Convention internationale sur les expositions, ratifiée en 1912 par seize États-membres, en dicte les bases légales ${ }^{11}$. Regroupés au sein d'une

7. Sur cette notion et la variété des phénomènes qu'elle recouvre: Thomas Grossbölting, "Im Reich der Arbeit». Die Repräsentation gesellschaftlicher Ordnung in den deutschen Industrie- und Gewerbeausstellungen 1790-1914, Munich, R. Oldenbourg Verlag, 2008, pp. 164-165.

8. Luc Rojas, "Les chambres de commerce, un organe de renseignement au service des industriels: l'exemple de la chambre de commerce de Saint-Étienne (1850-1930)", Histoire, économie E société, vol. IV, 2012, pp. 49 sq.

9. Georges Hantz, "Les arts décoratifs à l'Exposition de Milan ", Journal de Genève, n 124, 6 mai 1907, p. 2.

10. Sur la FICPE, ainsi que sur le cas suisse et la création de l'Office central suisse pour les expositions : Claire-Lise Debluë, Exposer pour exporter. Culture visuelle et expansion commerciale en Suisse (1908-1939), Neuchâtel, éd. Alphil, 2015.

11. L'Allemagne, l'Autriche, la Belgique, le Danemark, l'Espagne, la France, la Hongrie, l'Italie, le Japon, la Norvège, les Pays-Bas, le Portugal, le Royaume-Uni, la Russie, la Suède, et la Suisse ratifient la Convention en 1912. 
organisation centrale, les différents comités relaient auprès de leurs gouvernements respectifs les dispositions adoptées par la FICPE, afin de favoriser l'introduction d'une régulation des expositions dans les différentes législations nationales. Ces nouveaux acteurs collectifs de la politique d'exposition, généralement issus des organisations représentatives du commerce et de l'industrie, plébiscitent le principe de l'exposition internationale spécialisée. Si ce type d'expositions s'était multiplié dès les années 1870 de nombreuses manifestations sont encore organisées à la veille de la Première Guerre mondiale selon ce modèle, qu'il s'agisse de l'Exposition internationale d'Hygiène de Dresde en 1911 ou, la même année, de l'Exposition internationale des Industries et du Travail de Turin. Bien que l'exposition commerciale connaisse une forme de reconfiguration dès la Grande Crise qui marque le dernier tiers du XIX ${ }^{e}$ siècle, la Première Guerre mondiale accélère le processus et redéfinit ses orientations majeures.

\section{"EN FAVEUR DE LA GRANDE CAUSE DE L'EXPANSION ${ }^{12}$ " : \\ LA FOIRE D'ÉCHANTILLONS, UN MODĖLE TRANSNATIONAL ?}

L'éclatement de la guerre réduit en effet à néant les efforts entrepris depuis le début du siècle pour aboutir à une réglementation des expositions internationales. Sur le plan des relations commerciales et, plus particulièrement dans le domaine douanier, les dispositions tarifaires autrefois entérinées par les organisations membres de la FICPE sont balayées par les mesures protectionnistes de l'économie de guerre. L'entrée en vigueur de la Convention internationale ratifiée en 1912 par les membres de la FICPE est non seulement suspendue, mais le cycle des expositions internationales est, pour la première fois, brutalement interrompu. La crise du modèle libre-échangiste et les offensives protectionnistes menées par les grandes puissances contraignent les acteurs de la diplomatie commerciale à redéfinir leurs stratégies de conquête des marchés extérieurs ${ }^{13}$. Dans ce contexte, les institutions dédiées à la régulation des expositions perdent, en grande partie, leur raison d'être. Les services officiels attachés aux relations extérieures ou des chambres de commerce s'efforcent en revanche d'accroître et de diversifier leurs moyens d'action ${ }^{14}$. La foire dite moderne en constitue l'un des instruments privilégiés.

Contrairement à leur modèle historique, les foires de marchandises du Haut Moyen-Âge et de l'époque moderne, véritables «temps forts de la

12. Charles Touzot, "La Foire de Lyon. Instrument d'expansion française ", Lyon. Revue mensuelle d'expansion commerciale. Organe officiel de la Foire de Lyon, n 9, mars 1921, p. 28.

13. Ephraim Maisel, "The Formation of the Department of Overseas Trade, 1919-26 ", Journal of Contemporary History, $\mathrm{n}^{\circ} 1$, vol. XXIV, janvier 1989, pp. 171 sq.

14. Philippe Lacombrade, "L'organisation de la Chambre : son évolution de 1900 à 1939 ", in Paul Lenormand (dir.), La Chambre de commerce et d'industrie de Paris (1803-2003), Genève, Droz, 2008, p. 56. 
vie commerciale », qui se déroulaient dans les centres urbains situés sur les grands axes de circulation européens ${ }^{15}$, les foires d'échantillons proscrivent la vente directe. Appelées à se substituer aux expositions universelles dont le principe serait, estime-t-on, dépassé, celles-ci présentent les qualités d'une institution commerciale moderne. Outre leur vocation à lutter contre les obstacles protectionnistes aux échanges commerciaux, elles sont également destinées à favoriser les contacts entre producteurs et acheteurs, entre visiteurs et exposants et, de manière générale, à encourager les transactions commerciales grâce aux services dispensés par le bureau de renseignements de la foire et aux facilités douanières consenties par les gouvernements ${ }^{16}$. Les foires comportent également un volet financier qui vise à simplifier les échanges de devises et l'octroi de visas d'exportation ${ }^{17}$. Elles exercent par ailleurs une fonction de contrôle et de régulation des prix. Face à l'insécurité des relations financières et commerciales internationales et aux problèmes de ravitaillement ou d'écoulement des marchandises rencontrés par de nombreux secteurs économiques, les foires regroupent et concentrent, dans le temps et dans l'espace, les différents acteurs du négoce international. Le principe qui gouverne la foire est incontestablement celui de l'efficacité : "Le maximum d'affaires sur le minimum d'espace, dans le minimum de temps, avec le minimum de frais " résume ainsi un imprimé de la Foire de Lyon, selon la formule consacrée par son fondateur, le maire de la ville Édouard Herriot (Fig. 1).

Le caractère périodique des foires offre une grande souplesse dans le choix et la composition des groupes et permet aux acheteurs, comme aux exposants, de se rencontrer régulièrement, dans une atmosphère que l'on veut favorable à la conclusion d'affaires. En encourageant la présentation de nouveautés, elles fonctionnent comme un véritable «baromètre conjoncturel " qui offre aux acheteurs la possibilité d'évaluer l'état du marché ${ }^{18}$. Lors de leur visite, ceux-ci recueillent en effet de nombreux renseignements au sujet des produits apparus depuis peu sur le marché, comparent les différentes offres et prennent connaissance des prix en vigueur. Les foires constituent ainsi bien plus que de simples vitrines de la production nationale. Durant la guerre, les foires restent essentiellement dédiées au commerce, marquant une certaine distance avec les entreprises de divertissement de masse qu'avaient constitué les expositions universelles dans

15. Anne Radeff, "Faire les foires. Mobilités et commerce périodique dans l'ancien canton de Berne à l'époque moderne ", Bulletin du Centre Pierre Léon d'histoire économique et sociale, $\mathrm{n}^{\text {os }} 2-3-4$, 1992, pp. 67-83. Citation : Dominique Margairaz, "Institutions et espaces de marché : de l'abstrait au concret ", in Françoise Bayard, Patrick Fridenson, Albert Rigaudère (dir.), Genèse des marchés, Paris, Comité pour l'histoire économique et financière de la France, 2015, p. 60.

16. À Lyon, un service d'admission temporaire est ainsi mis en place pour la durée de la foire : Archives économiques suisses/Schweizerisches Wirtschaftsarchiv (SWA), C 138, "La Foire de Lyon du $1^{\text {er }}$ au 15 mars $1919 »$, p. 13.

17. Victoria de Grazia, Irresistible Empire. America's Advance through Twentieth-Century Europe, Cambridge/Londres, Belknap Press of Harvard University Press, 2005, p. 189.

18. Thomas Grossbölting, op. cit., pp. 82-83. 
la deuxième moitié du XIX ${ }^{\mathrm{e}}$ siècle. C'est ainsi en grande partie contre ce modèle que les foires d'échantillons s'efforcent de bâtir leur réputation.

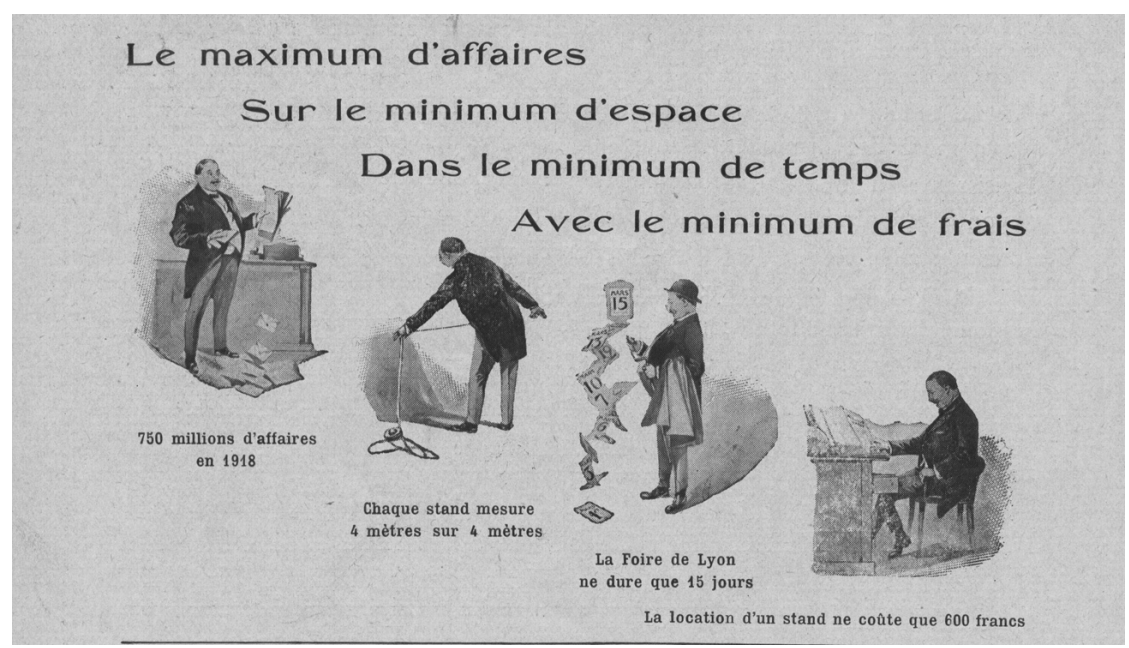

Fig. 1: "Foire de Lyon. La quatrième foire de guerre, du $1^{\text {er }}$ au 15 mars 1919 ", p. 14 (détail), Schweizerisches Wirtschaftsarchiv CH SWA Ausstellungen C 138

Firmendossier zur Foire internationale de Lyon.

\section{L'ESSOR DES FOIRES D'ÉCHANTILLONS : DE LA CONCURRENCE À LA MISE EN RÉSEAU}

Parmi les très nombreuses foires qui se développent au tournant de la Première Guerre mondiale, la Leipziger Messe est incontestablement celle qui jouit de la plus grande renommée. D'une ampleur remarquable - elle réunit lors de son édition du printemps 1917 plus de 2500 exposants elle est aussi la première institution de ce type à rompre avec le modèle de la foire de marchandises (Warenmesse) pour adopter, dès 1895, celui de la foire d'échantillons ${ }^{19}$. Jusqu'en 1914, la foire de Leipzig bénéficie d'une situation de monopole qui prend fin avec la création de plusieurs manifestations internationales. Entre 1915 et 1917, on compte ainsi non moins de cinq nouvelles foires d'échantillons, à Lyon ${ }^{20}$, Bordeaux, Londres, Bâle et Utrecht. À l'issue de la guerre, leur nombre s'accroît encore

19. H.[ermann] Behrmann, Führer durch die Leipziger Mustermessen, Herausgegeben vom Messamt für die Mustermessen in Leipzig, 2. Auflage, Juni 1918, Leipzig, éd. Julius Klinkhardt, 1918, pp. 23 sq. Sur les changements intervenus durant la guerre dans l'administration et l'organisation de la Foire de Leipzig : Jochen Geyer, «Die selbstverwaltete Messe. Der Messestandort Leipzig zwischen 1916 und 1945 ", in Hartmut Zwahr, Thomas Topfstedt, Günter Bentele (dir.), Leipzigs-Messen, 1497-1997, Cologne/Weimar/Vienne, Böhlau Verlag, vol. II, 1999, pp. 451-470.

20. Serge Berstein, Édouard Herriot ou la République en personne, Paris, Presses de la Fondation nationale des sciences politiques, 1985, pp. 50 sq. 
considérablement : "Il n'est pas jusqu'à l'île de Java qui n'ait organisé sa Foire annuelle d'Échantillons ", ironise ainsi au début des années 1920 un observateur suisse $\mathrm{e}^{21}$. La multiplication des foires entraine une inévitable mise en concurrence de ces différentes institutions commerciales. Pour y faire face, les promoteurs de la foire de Leipzig entreprennent notamment de développer son service de presse et de publicité et de moderniser ses institutions ${ }^{22}$. En 1916, sa direction administrative est regroupée au sein d'une structure centralisée dans laquelle les associations industrielles et commerciales obtiennent de nouvelles prérogatives aux dépens des autorités publiques. La Leipziger Messe connait alors une forte croissance qui lui permet de s'affirmer comme une institution économique moderne $e^{23}$.

Si des disparités existent, certes, entre les différentes foires, celles-ci résident essentiellement dans les détails de leur organisation et les déclinaisons multiples d'un modèle désormais transnational. Certaines foires d'échantillons, comme celles de Leipzig, de Lyon ou, plus tard, de Barcelone, sont internationales - bien qu'elles n'admettent en réalité, durant la guerre et dans l'immédiat après-guerre, que des exposants issus des pays alliés ou neutres ${ }^{24}-$, tandis que d'autres, comme Bâle ou Utrecht, ont un caractère national. Les manifestations qui, à l'instar de la foire de Leipzig, sont spécialisées, acceptent progressivement un nombre plus important de catégories de produits, généralement de consommation courante, afin d'élargir le cercle des acheteurs intéressés. Enfin, les modalités de l'intervention publique varient considérablement d'un cas à l'autre. Sans bénéficier de la tutelle officielle des États, elles jouissent, pour la plupart, d'importantes subventions publiques qui s'ajoutent au soutien financier dispensé par les chambres de commerce ou d'autres acteurs collectifs privés.

Les disparités observées et les rapports de concurrence qui régissent, durant la guerre, les relations entre ces différentes institutions commerciales ne doivent pas dissimuler le fait que celles-ci partagent en réalité une vision analogue de l'organisation économique et sociale, à une période où la légitimité du modèle libéral est violemment contestée ${ }^{25}$. La circulation de ce modèle rencontre d'ailleurs un tel succès qu'au "réseau transnational des expositions » décrit par l'historien Alexander G. Geppert à propos du long $\mathrm{XIX}^{\mathrm{e}}$ siècle ${ }^{26}$, se substitue un réseau transnational des foires,

21. L. G., op. cit., p. 27.

22. Grazyna-Maria Peter, "Messe-Werbung. Von der "Literarischen Abteilung" 1917 zur modernen Unternehmenskommunikation der Leipziger Messe 1997 ", in Hartmut Zwahr et al. (dir.), op. cit., pp. 704 sq.

23. Jochen Greyer, op. cit., pp. 453-454.

24. En 1916, la Foire internationale de Lyon exclut par exemple les produits issus d'Allemagne, d'Autriche, de Bulgarie et de Turquie: Schweizerisches Wirtschaftsarchiv, Bâle (ci-après : SWA), C 138, "Messe in Lyon, vom 1-15. März 1916 (Auszug der französischen Druckschrift) ", décembre 1915.

25. Charles S. Maier, Recasting Bourgeois Europe. Stabilization in France, Germany and Italy in the Decade after World War I, Princeton, Princeton University Press, 1975, pp. 5 sq.

26. Rien qu'en Allemagne, le nombre de foires passe de 10 à 112 entre 1919 et 1924, même si la majorité d'entre elles conservent un caractère essentiellement régional. Wolfram Fischer, "Zur 
qui s'institutionnalise, en 1925, à travers la fondation de l'Union des foires internationales (UFI). Sous les auspices de la Chambre de commerce internationale, l'UFI contribue non seulement à endiguer l'« hypertrophie » et la « fièvre des foires $\|^{27}$, mais également à rétablir un réseau international de l'expansion commerciale, voué à lutter contre les instabilités économiques et à favoriser l'ouverture de nouveaux débouchés ${ }^{28}$.

\section{LES DÉBUTS DE LA FOIRE NATIONALE SUISSE D'ÉCHANTILLONS : ÉCONOMIE DE GUERRE, «LUTTE CONTRE LA CONCURRENCE ÉTRANGÈRE » ET EXPANSION COMMERCIALE}

Malgré son statut d'État neutre, la Suisse ne reste pas en marge de ce mouvement transnational des foires. Les répercussions de la guerre sur les relations économiques extérieures de la Suisse sont en effet considérables, en particulier si l'on tient compte du fait qu'à la veille de la Première Guerre mondiale, le niveau des exportations par habitant se situait juste en dessous de celui de la Grande-Bretagne et de la Belgique ${ }^{29}$. La politique de blocus mise en place par les pays de l'Entente, dès 1915, dresse notamment d'importants obstacles à la circulation des marchandises et soustrait un nombre considérable de marchés à l'industrie d'exportation ${ }^{30}$. Sur le plan intérieur, l'inflation et les problèmes d'approvisionnement conduisent à une grave crise du ravitaillement et des ventes. Située sur une zone frontalière, en bordure du Rhin qui constitue alors une importante voie pour le transit des marchandises vers le nord de l'Europe, Bâle subit de plein fouet les effets de la guerre. De nombreuses voies de transit, notamment avec l'Allemagne, sont barrées, tandis que les contrôles aux frontières se multiplient ${ }^{31}$. C'est dans ce contexte que le projet d'une foire est évoqué pour la première fois par le directeur de l'École des arts et métiers de la ville de Bâle, Jules de Praetere. Conçu à l'origine comme un "marché " saisonnier au service des productions de " goût " et de "qualité ", le projet

Geschichte der Messen in Europa ", in Karl-Heinz Strohmann, Manfred Busche (dir.), Handbuch Messemarketing, Wiesbaden, Gabler Verlag, 1992, p. 11. Alexander C.T. Geppert, Fleeting Cities: Imperial Expositions in "Fin-de-siècle" Europe, Houndmills [etc.], Palgrave Macmillan, 2010, p. 3.

27. E. Rey, "Hypertrophie dans le domaine des foires et des expositions ", La Foire suisse d'échantillons, Bulletin officiel, $\mathrm{n}^{\circ} 10,1925$, p. 237.

28. Stephen Gross, "Selling Germany in South-Eastern Europe: Economic Uncertainty, Commercial Information and the Leipzig Trade Fair 1920-40 ", Contemporary European History, $n^{\circ} 1$, vol. XXI, février 2012, p. 23.

29. Thomas David, Bouda Etemad, "Les relations commerciales de la Suisse avec les Grandes Puissances durant l'entre-deux-guerres. Un survol chiffré ", in Sébastian Guex (dir.), La Suisse et les grandes puissances, 1914-1945, Genève, Droz, 1999, p. 31.

30. Patrick Halbeisen, "Wirtschaftspolik im "Zeitalter der Katastrophen” (1914-1945 ) ", in Patrick Halbeisen, Margrit Müller, Béatrice Veyrassat (dir.), Die Wirtschaftsgeschichte der Schweiz im 20. Jahrhundert, Bâle, Schwabe Verlag, 2012, p. 999.

31. Robert Labhardt, "Der Grenzraum Basel im Ersten Weltkrieg », Basler Zeitschrift für Geschichte und Alterumskunde, vol. CXIV, 2014, p. 51. 
prend rapidement une envergure plus importante, pour finalement s'inscrire parmi les mesures de l'économie de guerre ${ }^{32}$.

Le principe d'une foire nationale d'échantillons est approuvé au début de l'année 1916 par le gouvernement bâlois. Malgré l'important soutien financier consenti par les autorités cantonales, la foire conserve le statut d'une institution privée. La composition de son comité d'organisation témoigne de l'étroite collaboration établie entre l'État fédéral et l'économie dans le cadre de la Foire. On trouve à sa tête des acteurs appartenant à la puissante Chambre bâloise de commerce et d'industrie et à l'Association de la navigation sur le Rhin, des commerçants ou encore des représentants du secteur bancaire et de l'hôtellerie ${ }^{33}$. Quant à la présidence du comité, elle est confiée au chef de l'exécutif cantonal bâlois, le socialiste Hermann Blocher. Le rôle crucial conféré aux acteurs privés est emblématique des reconfigurations opérées dans le domaine de la politique d'exposition. Jusqu'alors en effet, l'Office central suisse pour les expositions (OCSE) et, avec lui, les trois plus importantes organisations patronales helvétiques bénéficiaient d'une situation de monopole. Créé en 1908, peu après la fondation de la FICPE, l'OCSE avait marqué une étape décisive pour l'institutionnalisation de la politique d'exposition et la professionnalisation d'un champ alors fort peu structuré ${ }^{34}$. Si l'OCSE avait résulté de la centralisation la politique d'exposition, la Foire de Bâle signale au contraire un mouvement de décentralisation favorable aux chambres cantonales de commerce. Ce phénomène se vérifie du reste une nouvelle fois, lors de la création du Comptoir suisse des industries alimentaires et agricoles de Lausanne en 1919 par la Chambre vaudoise de commerce et d'industrie.

Célébrée au printemps 1917, l'ouverture de la première foire suisse d'échantillons réunit plus de 800 exposants et enregistre, aux dires de ses organisateurs, près de 300000 entrées (Fig. 2). La foire se présente comme un instrument au service de l'économie nationale. Elle a pour vocation de stimuler l'exportation et de favoriser l'écoulement de la production sur le marché intérieur, tout en favorisant la création d'industries nouvelles. Dans les faits, la première édition de la manifestation vise essentiellement à développer le marché intérieur, non sans exalter le patriotisme économique des consommateurs. Malgré les efforts déployés par la direction, les représentants de l'industrie d'exportation restent longtemps minoritaires, allant même jusqu'à exprimer une ferme opposition au principe de la foire nationale ${ }^{35}$. Fortement tributaires de l'importation de matières premières et de produits semi-transformés, les entreprises tournées vers l'exportation combattent vigoureusement

32. SWA, PA 486, A 4, lettre de Jules de Praetere à la Nouvelle société helvétique, 15 juin 1915.

33. «Bericht über die I. Schweizer Mustermesse in Basel vom 15. Bis 29. April 1917. Beilage I zum Ratschlag betr. Finanzierung und Organisation der Schweizer Mustermesse in Basel. Dem Grossen Rat des Kantons Basel-Stadt vorgelegt den 10. Oktober 1918 ", [s.d.], [s.n.],[s.1.], pp. 27-28.

34. Claire-Lise Debluë, op. cit.

35. A. Haas, "Messe und Propaganda ", L'Exportateur suisse, n 5, vol. IX, mai 1923, p. 69. 
les mesures protectionnistes ${ }^{36}$. Ce n'est ainsi qu'à partir du milieu des années 1920, alors que la foire de Bâle s'apparente de plus en plus à une "foire d'exportation" (Exportmesse), que l'on observe un changement notoire dans la composition des groupes représentés.

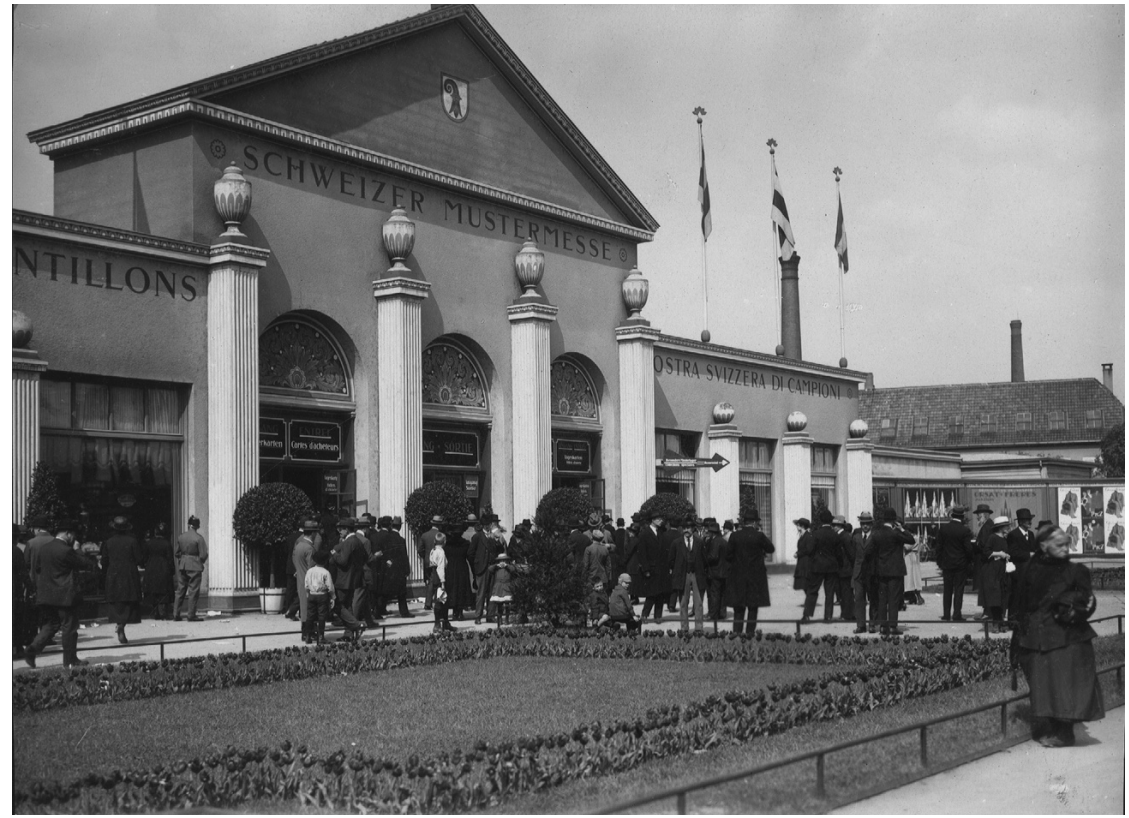

Fig. 2 : Entrée principale de la halle provisoire de la Foire suisse d'échantillons à Bâle, c. 1917-1923. CH SWA, H + I D 361 Firmendossier zur Genossenschaft Schweizer Mustermesse.

Parmi les nombreuses foires créées au cours de cette période, la Foire de Bâle fait pour ainsi dire figure d'exception. Définie comme une foire nationale, sa direction n'admet, en principe, aucun produit d'origine étrangère. En période de guerre, celle-ci justifie un tel choix par la nécessité de maintenir un strict devoir de neutralité. Le modèle auquel se réferrent les promoteurs de la foire n'est alors pas inédit. Lors des expositions nationales qui s'étaient déroulées successivement à Zurich, Genève et Berne entre 1883 et 1914, l'interdiction des produits étrangers avait été prononcée à chaque fois. S'il s'agissait alors de ménager les intérêts de l'industrie d'exportation et des milieux protectionnistes, les promoteurs de la foire d'échantillons de Bâle parviennent à faire de ce principe un gage de la neutralité économique de la Suisse. La proscription des produits étrangers répond en effet aux pressions exercées par les pays belligérants sur les Etats neutres. L'Entente avait introduit notamment, dès le mois de mars 1915,

36. Margrit Müller, "Internationalisierung trotz weltwirtschaftlicher Desintegration und vielfältiger Handeslbarrieren : erste Hälfte des 20. Jahrunderts ", in Patrick Halbeisen, Margrit Müller, Béatrice Veyrassat (dir.), Die Wirtschaftsgeschichte der Schweiz im 20. Jahrhundert, Bâle, Schwabe Verlag, 2012, p. 369. 
une série de mesures de contrôle sur les produits issus des neutres et exige des exportateurs suisses qu'ils présentent un certificat attestant de l'origine des marchandises. Dans ce contexte, l' "internationalisation " de la foire, ainsi qu'on la désigne alors, est exclue ${ }^{37}$. Pour les promoteurs de la foire de Bâle qui s'efforcent de réunir un nombre important d'exposants, il est du reste indispensable de distinguer leur entreprise du modèle historique de l'exposition nationale :

La Foire d'échantillons est une fête du travail et non pas du plaisir [...]. Le succès moral et économique de la foire sur l'exposition est évident. L'exposition nationale des temps nouveaux pour l'homme nouveau est là : la foire d'échantillons à Bâle ${ }^{38}$.

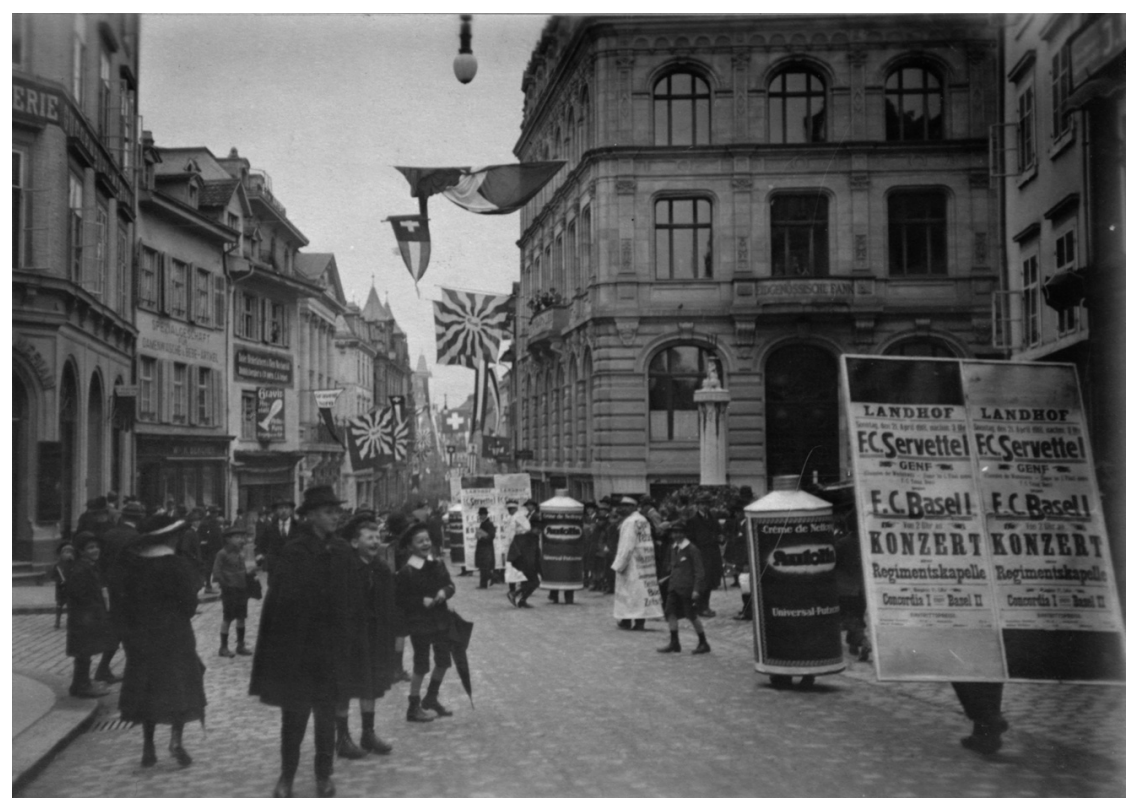

Fig. 3 : «Hommes réclame » dans la rue, durant la foire de Bâle, 1918.

Staatsarchiv Basel-Stadt, AL 45, 5-73-6.

Pour le public professionnel, la foire présente de précieux avantages. Outre les réductions accordées sur les billets de train, un service de logement offrant la possibilité de séjourner à proximité de la manifestation est mis à la disposition des visiteurs. Afin de " gagner un temps précieux pour

37. «Der Anspruch Basels auf die Schweizerische Mustermesse. Zur Vorgeschichte der Schweizerischen Mustermesse ", 5 mars 1918, SWA, H + I D 361.

38. "Die Mustermesse ist ein Fest der Arbeit, nicht des Vergnügens ; deren Besuch und Erledigung ist konzentrierte Aufmerksamkeit. Der moralische und ökonomische Erfolg der Messe gegenüber der Ausstellung liegt auf der Hand. [...] Die Landesausstellung der neuen Zeit für neue Menschen ist da : Die Mustermesse in Basel.", in Werner Zogg, "Mustermesse und Ausstellung ", La Foire suisse d'échantillons. Bulletin officiel, $\mathrm{n}^{\circ} 7$, 22 avril 1920, p. 299 (traduction de l'auteure). 
la conclusion des affaires à la Foire ", le public professionnel est invité à préparer minutieusement sa visite en s'aidant du catalogue publié dans les semaines précédant la manifestation ${ }^{39}$. La stricte limitation des frais engagés dans la foire et l'organisation méthodique de la manifestation ainsi que l'important service de renseignements mis à la disposition des acheteurs constituent les atouts incontestables d'une telle institution commerciale. À partir de 1918, des cabines aménagées sur le stand même permettent au vendeur et à son client de converser à l'abri des regards indiscrets, "en toute tranquillité et à toute heure ${ }^{40} »$. Si, dans l'enceinte de la foire, la vente directe est proscrite, le carnet de commandes y est roi.

L'effort de distinction des foires vis-à-vis des expositions internationales ou universelles se manifeste également, dès 1918, à travers une réglementation plus stricte de l'accès à la manifestation. Des cartes d'acheteurs sont ainsi distribuées aux clients professionnels qui bénéficient d'horaires spéciaux pour visiter la foire. La restriction des horaires de visite du grand public a pour principal objectif de limiter l'afflux des badauds. Bien que ces derniers ne soient théoriquement autorisés à se rendre sur les lieux de la manifestation que durant les après-midi, les samedis et les dimanches, les premières éditions de la foire accueillent un nombre très important de visiteurs non professionnels, suscitant le mécontentement des acheteurs et, davantage encore, des exposants. Le fait est intéressant, car il montre qu'en dépit des ambitions affichées par leurs organisateurs, les premières foires n'ont pas toujours la faculté de se distinguer véritablement des expositions. Les foires demeurent, il est vrai, des événements populaires. Défilés, dégustations et concerts rythment dix jours durant la manifestation, tandis que les hommes-sandwichs et les "porteurs d'affiches-réclames " envahissent les rues du centre-ville (Fig. 3). À une période cruciale pour l'institutionnalisation et la professionnalisation du champ publicitaire ${ }^{41}$, la foire s'impose non seulement comme un "laboratoire d'affaires ", mais également comme un lieu d'expérimentation des pratiques promotionnelles, autrement dit, comme "un foyer de propagande et de réclame " ${ }^{42}$. C'est ainsi à travers la reconnaissance de sa "valeur publicitaire " que la foire apportera la preuve de son efficacité commerciale ${ }^{43}$.

DU STAND À L'IMPRIMÉ : LA FOIRE D’ÉCHANTILLONS COMME MODÈLE D'ORGANISATION COMMERCIALE

Les reconfigurations observées ne se limitent pas à l'organisation économique de la foire, mais dictent également de nouvelles dispositions en

39. "Il faut préparer la visite de la Foire ", Bulletin officiel de la Foire suisse, n 3, mars 1930 p. 73.

40. Rapport sur la Foire suisse d'échantillons à Bâle 1918 (15-30 avril), [s.l.] [s.d.] p. 1.

41. Marie-Emmanuelle Chessel, op. cit., pp. 20 sq. Sur le cas suisse : Claire-Lise Debluë, op. cit.

42. Pour les deux citations, respectivement : L. G., op. cit., p. 27, et E. Rey, op. cit., p. 280.

43. Karl Lauterer, Der Werbewert der Mustermessen, Bâle, B. Schwabe, 1922. 
matière de réclame et de communication commerciale, de l'aménagement des stands à la conception formelle des imprimés ou des périodiques. Si les expositions universelles avaient accoutumé les exposants à rivaliser d'ingéniosité dans la conception de leurs aménagements, les foires prétendent, au contraire, les libérer de telles contraintes. On estime ainsi que le prestige d'une maison ne se mesure pas à l'audace de sa décoration ou à la richesse de ses aménagements, mais à la qualité de ses échantillons. En période de crise, et pour rompre une nouvelle fois avec le faste si décrié des expositions universelles, la rigueur et la sobriété sont conçues comme un indicateur du sérieux de l'entreprise. Ainsi préconise-t-on dans le bulletin de la foire :

Dans les salles de la Foire centrale, les échantillons, ordonnés simplement, se détacheront bien les uns des autres afin que l'on puisse les examiner des yeux et des doigts, cela dans le but d'épargner toute hésitation à l'acheteur pressé, car il ne s'est pas dérangé à l'intention d'objets dont la vue est inutilement obstruée sous des vitrines ou des pavillons décoratifs. Que l'on tienne compte des intérêts de l'acheteur en regard de ceux du vendeur et l'on se convaincra que l'objectivité la plus absolue est de première nécessitét ${ }^{44}$.

L'attitude du représentant de commerce elle-même est soigneusement décomposée afin que celui-ci serve au mieux les intérêts de la maison qui le mandate.

La professionnalisation de la pratique des expositions s'observe donc sur les lieux mêmes de la manifestation. Dans les différentes publications périodiques éditées à l'occasion, il n'est pas rare que les conseils aux exposants s'apparentent à un véritable programme de rénovation des méthodes commerciales, dont la foire constituerait l'élément clé. Si l'on estime que les frais entraînés par les installations doivent être réduits au minimum, tout en présentant les attributs d'un étalage de qualité, l'application de ces recommandations ne va pas de soi. Bien peu d'exposants bénéficient en effet de l'expérience requise pour se conformer aux prétendues normes esthétiques d'une foire moderne. Les publications officielles ont une fonction didactique essentielle. Dans le bulletin de la Foire, les conseils dispensés aux exposants profanes sont monnaie courante et empruntent volontiers le langage de la psychologie commerciale. Qu'il soit question de la conception des stands ou de la forme des imprimés, la nécessité de frapper l'attention du visiteur est en effet au cœur de la stratégie commerciale. "La peine prise pour cela n'est jamais prise en vain ", assure un rédacteur du Bulletin officiel de la Foire suisse ${ }^{45}$.

Des «stands-modèles » dédiés aux exposants souhaitant affiner leurs compétences en matière d' "étalage ", sont aménagés sur les lieux mêmes

44. Alfred Ditisheim, "Étude sur la manière de présenter les modèles à la Foire suisse d'échantillons ", La Foire suisse d'échantillons, n 3, 15 décembre 1916, p. 72.

45. "Préparatifs des participants à la Foire Suisse d'Échantillons ", La Foire suisse d'échantillons Bâle. Bulletin officiel, $\mathrm{n}^{\circ}$ 1, janvier 1919, p. 2, [s.n.]. 
de la foire et reproduits dans le bulletin ${ }^{46}$. Des cours de décoration sont même dispensés aux éventuels intéressés. La construction d'un stand conforme aux prescriptions de la direction constitue un gage de professionnalisme indispensable au renom de la manifestation : "La simplicité, l'aspect agréable et la clarté doivent être ses principes directeurs ", préconise ainsi l'un des rédacteurs du Bulletin ${ }^{47}$. Dans d'autres cas, lorsque le stand excède une certaine surface, le recours à un décorateur est en revanche vivement conseillé. Le caractère périodique de la foire permet à ses participants de nouer des contacts privilégiés avec certains décorateurs de la place bâloise qui, année après année, dispensent leurs services aux exposants. L'aménagement des stands est ainsi conçu comme une véritable vitrine de la modernité commerciale.

Si le processus de rationalisation commerciale se manifeste notamment dans la manière de définir de nouvelles normes en matière d'" étalagisme ", les publications périodiques font elles aussi l'objet d'une organisation rigoureuse. Le fait n'est pas propre à la foire de Bâle, puisque d'autres institutions analogues possèdent leur propre service de presse ${ }^{48}$. La politique éditoriale de la foire constitue un élément clé de la stratégie commerciale de l'entreprise. L'entretien des relations d'affaires se déploie dans le temps et dans l'espace, bien au-delà des quelques jours durant lesquels se déroule la manifestation :

Le succès de la Foire ne réside pas seulement dans la représentation, dans l'exposition des marchandises ou dans la présence personnelle pendant les $10 \mathrm{ou}$ 15 jours que dure la Foire. Il présuppose plutôt, avant et après la Foire, un travail systématique et bien combiné, la possession d'une bonne liste d'adresses à compléter pendant la Foire, absolument nécessaire pour permettre d'éveiller l'intérêt et de nouer des connaissances personnelles ${ }^{49}$.

La durée limitée de la foire (de quelques jours à une ou deux semaines contre quatre à six mois pour une exposition universelle) contribue, il est vrai, à la constitution d'un solide réseau d'affaires. Elle permet notamment aux entreprises de mandater un représentant professionnel sur les lieux de la manifestation, pour un montant jugé raisonnable ${ }^{50}$. D'une édition à l'autre, différents types de publications rythment la vie commerciale, sans oublier les innombrables courriers, souvenirs et cadeaux envoyés aux différents clients. À côté du catalogue de la foire, qui offre un répertoire complet des entreprises et des produits présentés à Bâle, le bulletin mensuel de la manifestation constitue un instrument clé pour entretenir les relations

46. Alfred Ditisheim, op. cit., p. 71.

47. "Préparatifs des participants à la Foire suisse d'échantillons ", La Foire suisse d'échantillons Bâle. Bulletin officiel, $\mathrm{n}^{\circ}$ 1, janvier 1919, p. 3, [s.n.].

48. En 1921, la foire de Leipzig crée par exemple son propre service de presse. Grazyna-Maria Peter, op. cit., p. 704.

49. Karl Lauterer, "Après la Foire ", La Foire suisse d'échantillons. Supplément de l'Exportateur suisse, $\mathrm{n}^{\circ} 10,15$ juin 1923 , p. 3 .

50. Ed.[uard] Boos-Jegher, "Märkte, Messen, Ausstellungen », La Foire suisse d'échantillons. Bulletin officiel, $\mathrm{n}^{\circ}$ 6, 24 avril 1918, p. 126. 
nouées dans le cadre de la manifestation. Publié en allemand et en français, il s'adresse aussi bien aux visiteurs indigènes qu'étrangers. Les fabricants en quête de matières premières peuvent s'y référer et l'utiliser comme un répertoire industriel, consignant les différentes offres et les tarifs spéciaux pratiqués par certains producteurs. L'inflation du nombre d'imprimés et de circulaires diffusés dans le cadre de la foire de Bâle témoigne de l'effort considérable déployé dans le domaine de l'information commerciale. C'est ainsi que les enquêtes menées par leurs bureaux de renseignements sur la foi des informations recueillies auprès des acheteurs et des exposants contribuent à consacrer un nouveau type d'expertise économique, fondé sur l'étude statistique et les méthodes d'analyse de marché, qui connaissent une fortune certaine dans les années 1920 et $1930^{51}$.

\section{CONCLUSION}

Dès 1914, le principe d'une entente internationale autour des expositions se heurte aux impératifs de la "guerre totale ". Pour les contemporains, le déclin du modèle historique de l'exposition universelle semble alors inéluctable. Longtemps cité en exemple, le modèle de la Great Exhibition of All Nations perd le prestige dont elle avait longtemps joui, au profit de la Leipziger Messe qui devient le parangon d'une conception moderne des échanges commerciaux. De manière générale, les foires inaugurent une nouvelle ère dans la gestion des expositions, plus professionnalisée et davantage centralisée. Bien qu'une telle tendance fût déjà manifeste au sein de la FICPE et de ses différents comités nationaux, le phénomène s'accentue durant la Première Guerre mondiale.

$\mathrm{Au}$-delà de la fonction strictement commerciale assignée aux foires dans le cadre de l'économie de guerre, la distinction de principe établie entre les différentes déclinaisons de l'exposition tend pourtant à s'effacer progressivement. En Suisse, la création de l'Office suisse d'expansion commerciale en 1927 joue un rôle majeur dans ces nouvelles reconfigurations des pratiques de l'exposition. Au cours des années 1930, l'OSCE parvient non seulement à gagner un nombre important d'exposants au principe de la représentation collective, mais s'efforce également de s'attirer les faveurs du grand public, tournant le dos à certains des principes fondateurs des foires commerciales issues de la Première Guerre mondiale. Loin d'un retour aux formes antérieures du "spectacle industriel ", on assiste alors à une réassignation des expositions et des foires commerciales à un rôle plus largement politique et culturel. Avec la collaboration d'autres acteurs

51. Pour le cas français : Alain Chatriot, "La Chambre face à une institution nouvelle : le Conseil national économique (1925-1940)", in Christophe Bouneau, Paul Lenormand (dir.), La Chambre de commerce et d'industrie de Paris (1803-2003), Genève, Droz, 2008, pp. 75-98 ; Ludovic Tournès, "L'Institut scientifique de recherches économiques et sociales et les débuts de l'expertise économique en France (1933-1940)", Genèses, n 65, 2006, vol. IV, pp. 49-70. 
collectifs issus de l'industrie touristique ou du champ culturel, l'OSCE contribue ainsi à désigner les expositions et leurs institutions comme un instrument privilégié du rapprochement entre les acteurs de la diplomatie économique et culturelle.

Claire-Lise DebluË

Université de Lausanne 